\title{
Pan-lysyl oxidase inhibitor PXS-5505 ameliorates multiple-organ fibrosis by inhibiting collagen crosslinks in rodent models of systemic sclerosis
}

\author{
Yimin Yao $^{1}$, Alison Findlay ${ }^{1}$, Jessica Stolp ${ }^{1}$, Benjamin Rayner ${ }^{2}$, Kjetil Ask ${ }^{3}$, and Wolfgang \\ Jarolimek $^{1}$ \\ ${ }^{1}$ Pharmaxis Ltd \\ ${ }^{2}$ Childrens Cancer Institute of Australia for Medical Research \\ ${ }^{3}$ McMaster University
}

December 2, 2021

\begin{abstract}
Background/Purpose: Systemic sclerosis (SSc) is characterised by progressive multiple-organ fibrosis leading to morbidity and mortality. Lysyl oxidases play a vital role in the cross-linking of collagens and subsequent build-up of fibrosis in the extracellular matrix. As such, their inhibition provides a novel treatment paradigm for SSc. Experimental Approach: Lysyl oxidases are upregulated in preclinical models of fibrosis in skin, lung, heart, kidney and liver. A novel small molecule pan-lysyl oxidase inhibitor, PXS-5505, currently in clinical development for bone fibrosis treatment was evaluated in in vivo rodent models resembling the fibrotic conditions in SSc. Key Results: Both lysyl oxidase and lysyl oxidase-like 2 (LOXL2) expression was elevated in the skin and lung of SSc patients. Once-a-day oral application of PXS-5505 inhibited lysyl oxidase activity in the skin and LOXL2 activity in the lung. PXS-5505 exhibited anti-fibrotic effects in the SSc skin mouse model, reducing dermal thickness and $\alpha$-smooth muscle actin compared to the disease controls. Similarly, in the bleomycin-induced mouse lung model, PXS-5505 reduced tissue fibrosis toward normal levels. The anti-fibrotic efficacy of PXS-5505 in the bleomycin exposed lungs was mediated by its ability to normalise collagen/elastin crosslink formation, a direct consequence of lysyl oxidase inhibition. PXS-5505 also reduced area of fibrosis in rodent models of the ischaemia-reperfusion heart, the unilateral ureteral obstruction kidney and the CCl4-induced fibrotic liver. Conclusion/Implication: PXS-5505 consistently demonstrates potent anti-fibrotic efficacy in multiple models of organ fibrosis relevant to the pathogenesis of SSc, suggesting that it may be efficacious as a novel approach for treating SSc.
\end{abstract}

\section{Hosted file}

PXS-5505 and SSc.pdf available at https://authorea.com/users/449257/articles/547815-panlysyl-oxidase-inhibitor-pxs-5505-ameliorates-multiple-organ-fibrosis-by-inhibitingcollagen-crosslinks-in-rodent-models-of-systemic-sclerosis 